\title{
Erratum
}

\section{Doppler effect on gamma-rays bursts in the fireball framework}

\author{
Y.-P. Qin ${ }^{1}$ and F.-W. Zhang ${ }^{2}$ \\ 1 National Astronomical Observatories/Yunnan Observatory, Chinese Academy of Sciences, PO Box 110, Kunming, Yunnan, \\ 650011, PR China \\ 2 Physics Department, Guangxi University, Nanning, Guangxi, 530004, PR China
}

A\&A, 396, 705-713 (2002), DOI:10.1051/004-6361:20021439

Key words. gamma rays: bursts - gamma rays: theory - radiation mechanisms: non thermal - relativity - errata, addenda

In the paper, "Doppler effect of gamma-ray bursts in the fireball framework" by Y.-P. Qin (2002, A\&A, 396, 705, Paper I), the calculation in extreme cases such as $\Gamma=10000$ is very sensitive to the step of the integral over the fireball surface. Due to the slightly larger step of the integral, we obtained smaller values of some quantities when taking $\Gamma \geq 5000$. Based on a more precise calculation, the following correction should be made:

Tables 1-4, Figs. 5, and 10-13 in Paper I should be replaced by Tables 1-4 and Figs. 1-5 given here.

On page 708, "We find from these tables that $v_{\mathrm{p}}$ as well as $\left(v f_{v}\right)_{\mathrm{p}}$ rise with respect to increase of $\Gamma$ with a certain range (say, $\Gamma<5000$ ). Beyond some extent of $\Gamma$ (say, $\Gamma=10000$ ), while $\left(v f_{v}\right)_{\mathrm{p}}$ would continue to be larger, $v_{\mathrm{p}}$ becomes smaller." should be replaced by "We find from these tables that $v_{\mathrm{p}}$ as well as $\left(v f_{v}\right)_{\mathrm{p}}$ always rise with increasing $\Gamma$ ".

On page 710, "Only when the expansion speed is extremely large (say, $\Gamma=10000$ ), one can expect an increase in fluxes with respect to the decrease in the speed." should be omitted.

On page 710, "Also, only when the expansion speed is extremely large (say, $\Gamma=10000$ ), one can expect an increase in fluxes with respect to the decrease in the speed, which would also be rare due to the same reason." should be omitted.

On page 711, "Only when the expansion speed is extremely large (say, $\Gamma=10000$ ), one can expect an increase in fluxes with respect to the decrease in the speed for the above models." should be omitted.
Table 1. List of $v_{\mathrm{p}}$ and $\left(v f_{v}\right)_{\mathrm{p}}$ for the bremsstrahlung radiation.

\begin{tabular}{ccc}
\hline \hline$\Gamma$ & $v_{\mathrm{p}} / v_{0, \mathrm{~B}}$ & $\left(v f_{v, \mathrm{~B}}\right)_{\mathrm{p}}$ \\
\hline $1 \times 10^{0}$ & $9.99 \times 10^{-1}$ & $1.84 \times 10^{-1}$ \\
$2 \times 10^{0}$ & $3.06 \times 10^{0}$ & $1.16 \times 10^{2}$ \\
$5 \times 10^{0}$ & $8.00 \times 10^{0}$ & $3.48 \times 10^{4}$ \\
$1 \times 10^{1}$ & $1.62 \times 10^{1}$ & $2.29 \times 10^{6}$ \\
$2 \times 10^{1}$ & $3.23 \times 10^{1}$ & $1.47 \times 10^{8}$ \\
$5 \times 10^{1}$ & $8.09 \times 10^{1}$ & $3.60 \times 10^{10}$ \\
$1 \times 10^{2}$ & $1.62 \times 10^{2}$ & $2.31 \times 10^{12}$ \\
$2 \times 10^{2}$ & $3.25 \times 10^{2}$ & $1.48 \times 10^{14}$ \\
$5 \times 10^{2}$ & $8.08 \times 10^{2}$ & $3.60 \times 10^{16}$ \\
$1 \times 10^{3}$ & $1.62 \times 10^{3}$ & $2.31 \times 10^{18}$ \\
$2 \times 10^{3}$ & $3.24 \times 10^{3}$ & $1.48 \times 10^{20}$ \\
$5 \times 10^{3}$ & $8.12 \times 10^{3}$ & $3.60 \times 10^{22}$ \\
$1 \times 10^{4}$ & $1.62 \times 10^{4}$ & $2.31 \times 10^{24}$ \\
\hline
\end{tabular}

Table 2. List of $v_{\mathrm{p}}$ and $\left(v f_{v}\right)_{\mathrm{p}}$ for the Comptonized radiation.

\begin{tabular}{ccc}
\hline \hline$\Gamma$ & $v_{\mathrm{p}} / v_{0, \mathrm{C}}$ & $\left(v f_{v, \mathrm{C}}\right)_{\mathrm{p}}$ \\
\hline $1 \times 10^{0}$ & $1.40 \times 10^{0}$ & $1.97 \times 10^{-1}$ \\
$2 \times 10^{0}$ & $4.30 \times 10^{0}$ & $1.24 \times 10^{2}$ \\
$5 \times 10^{0}$ & $1.13 \times 10^{1}$ & $3.71 \times 10^{4}$ \\
$1 \times 10^{1}$ & $2.27 \times 10^{1}$ & $2.44 \times 10^{6}$ \\
$2 \times 10^{1}$ & $4.56 \times 10^{1}$ & $1.57 \times 10^{8}$ \\
$5 \times 10^{1}$ & $1.14 \times 10^{2}$ & $3.84 \times 10^{10}$ \\
$1 \times 10^{2}$ & $2.28 \times 10^{2}$ & $2.46 \times 10^{12}$ \\
$2 \times 10^{2}$ & $4.55 \times 10^{2}$ & $1.57 \times 10^{14}$ \\
$5 \times 10^{2}$ & $1.14 \times 10^{3}$ & $3.84 \times 10^{16}$ \\
$1 \times 10^{3}$ & $2.28 \times 10^{3}$ & $2.46 \times 10^{18}$ \\
$2 \times 10^{3}$ & $4.54 \times 10^{3}$ & $1.57 \times 10^{20}$ \\
$5 \times 10^{3}$ & $1.14 \times 10^{4}$ & $3.84 \times 10^{22}$ \\
$1 \times 10^{4}$ & $2.28 \times 10^{4}$ & $2.46 \times 10^{24}$ \\
\hline
\end{tabular}

Send offprint requests to: F.-W. Zhang,

e-mail: fwzhang@hotmail.com 
Table 3. List of $v_{\mathrm{p}}$ and $\left(v f_{v}\right)_{\mathrm{p}}$ for the synchrotron radiation.

\begin{tabular}{ccc}
\hline \hline$\Gamma$ & $v_{\mathrm{p}} / v_{0, \mathrm{~S}}$ & $\left(v f_{v, \mathrm{~S}}\right)_{\mathrm{p}}$ \\
\hline $1 \times 10^{0}$ & $3.68 \times 10^{3}$ & $1.68 \times 10^{4}$ \\
$2 \times 10^{0}$ & $1.13 \times 10^{4}$ & $1.07 \times 10^{7}$ \\
$5 \times 10^{0}$ & $2.99 \times 10^{4}$ & $3.19 \times 10^{9}$ \\
$1 \times 10^{1}$ & $6.00 \times 10^{4}$ & $2.10 \times 10^{11}$ \\
$2 \times 10^{1}$ & $1.21 \times 10^{5}$ & $1.35 \times 10^{13}$ \\
$5 \times 10^{1}$ & $3.00 \times 10^{5}$ & $3.31 \times 10^{15}$ \\
$1 \times 10^{2}$ & $6.03 \times 10^{5}$ & $2.12 \times 10^{17}$ \\
$2 \times 10^{2}$ & $1.20 \times 10^{6}$ & $1.36 \times 10^{19}$ \\
$5 \times 10^{2}$ & $3.01 \times 10^{6}$ & $3.31 \times 10^{21}$ \\
$1 \times 10^{3}$ & $6.01 \times 10^{6}$ & $2.12 \times 10^{23}$ \\
$2 \times 10^{3}$ & $1.20 \times 10^{7}$ & $1.36 \times 10^{25}$ \\
$5 \times 10^{3}$ & $3.01 \times 10^{7}$ & $3.31 \times 10^{27}$ \\
$1 \times 10^{4}$ & $6.00 \times 10^{7}$ & $2.12 \times 10^{29}$ \\
\hline
\end{tabular}

Table 4. List of $v_{\mathrm{p}}$ and $\left(v f_{v}\right)_{\mathrm{p}}$ for the GRB radiation.

\begin{tabular}{ccc}
\hline \hline$\Gamma$ & $v_{\mathrm{p}} / v_{0, \mathrm{P}}$ & $\left(v f_{v, \mathrm{G}}\right)_{\mathrm{p}}$ \\
\hline $1 \times 10^{0}$ & $9.99 \times 10^{-1}$ & $1.84 \times 10^{-1}$ \\
$2 \times 10^{0}$ & $3.13 \times 10^{0}$ & $1.17 \times 10^{2}$ \\
$5 \times 10^{0}$ & $8.28 \times 10^{0}$ & $3.50 \times 10^{4}$ \\
$1 \times 10^{1}$ & $1.66 \times 10^{1}$ & $2.30 \times 10^{6}$ \\
$2 \times 10^{1}$ & $3.34 \times 10^{1}$ & $1.48 \times 10^{8}$ \\
$5 \times 10^{1}$ & $8.33 \times 10^{1}$ & $3.62 \times 10^{10}$ \\
$1 \times 10^{2}$ & $1.67 \times 10^{2}$ & $2.32 \times 10^{12}$ \\
$2 \times 10^{2}$ & $3.34 \times 10^{2}$ & $1.48 \times 10^{14}$ \\
$5 \times 10^{2}$ & $8.33 \times 10^{2}$ & $3.62 \times 10^{16}$ \\
$1 \times 10^{3}$ & $1.67 \times 10^{3}$ & $2.32 \times 10^{18}$ \\
$2 \times 10^{3}$ & $3.34 \times 10^{3}$ & $1.48 \times 10^{20}$ \\
$5 \times 10^{3}$ & $8.33 \times 10^{3}$ & $3.62 \times 10^{22}$ \\
$1 \times 10^{4}$ & $1.67 \times 10^{4}$ & $2.32 \times 10^{24}$ \\
\hline
\end{tabular}

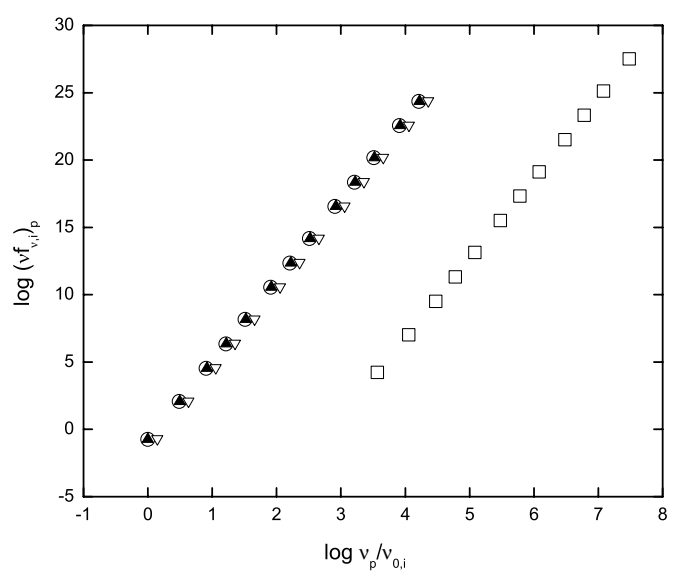

Fig. 1. The plots of $\log \left(v f_{v}\right)_{\mathrm{p}}-\log v_{\mathrm{p}}$ for the bremsstrahlung (open circle), Comptonized (open triangle), synchrotron (open square), and the GRB model (filled triangle) radiation, where $\log \left(v f_{v, i}\right)_{\mathrm{p}}$ represents $\log \left(v f_{v, \mathrm{~B}}\right)_{\mathrm{p}}, \log \left(v f_{v, \mathrm{C}}\right)_{\mathrm{p}}, \log \left(v f_{v, \mathrm{~S}}\right)_{\mathrm{p}}$ and $\log \left(v f_{v, \mathrm{G}}\right)_{\mathrm{p}}$, while $\log \left(v_{\mathrm{p}} / v_{0, i}\right)$ represents $\log \left(v_{\mathrm{p}} / v_{0, \mathrm{~B}}\right), \log \left(v_{\mathrm{p}} / v_{0, \mathrm{C}}\right), \log \left(v_{\mathrm{p}} / v_{0, \mathrm{~S}}\right)$ and $\log \left(v_{\mathrm{p}} / v_{0, \mathrm{P}}\right)$. The data are taken from Tables $1-4$.



Fig. 2. The plot of $\log \left(v f_{v, \mathrm{~B}}\right)-\log \Gamma$ for the bremsstrahlung radiation at various frequencies (the solid lines from the bottom to the top: $\log \left(v / v_{0, \mathrm{~B}}\right)=-4,-2,0$; the dashed line: $\log \left(v / v_{0, \mathrm{~B}}\right)=2$; the dotted line: $\log \left(v / v_{0, \mathrm{~B}}\right)=3$; the dashed and dotted line: $\log \left(v / v_{0, \mathrm{~B}}\right)=4$; the dashed dotted dotted line: $\left.\log \left(v / v_{0, \mathrm{~B}}\right)=5\right)$.

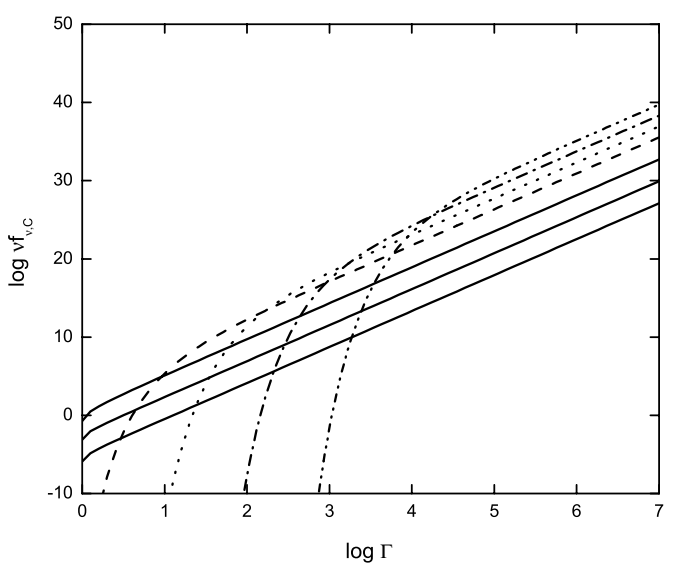

Fig. 3. The plot of $\log \left(v f_{v, \mathrm{C}}\right)-\log \Gamma$ for the Comptonized radiation at various frequencies (the solid lines from the bottom to the top: $\log \left(v / v_{0, \mathrm{C}}\right)=-4,-2,0$; the dashed line: $\log \left(v / v_{0, \mathrm{C}}\right)=2$; the dotted line: $\log \left(v / v_{0, \mathrm{C}}\right)=3$; the dashed and dotted line: $\log \left(v / v_{0, \mathrm{C}}\right)=4$; the dashed dotted dotted line: $\left.\log \left(v / v_{0, \mathrm{C}}\right)=5\right)$.

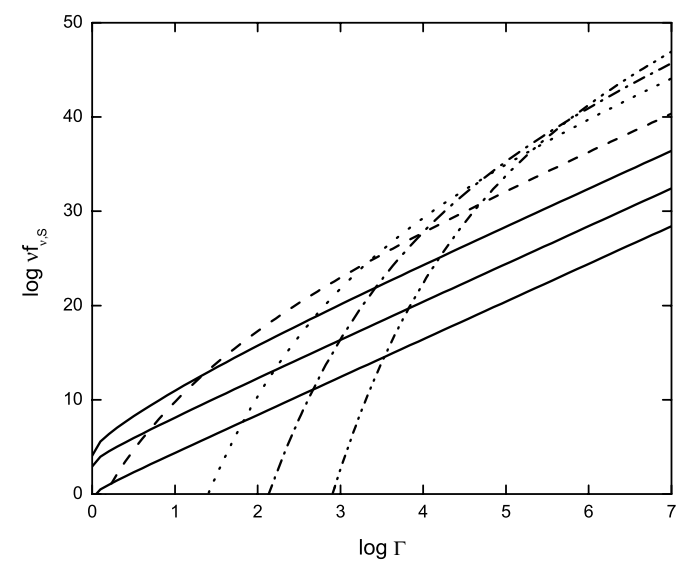

Fig. 4. The plot of $\log \left(v f_{v, \mathrm{~S}}\right)-\log \Gamma$ for the synchrotron radiation at various frequencies (the solid lines from the bottom to the top: $\log \left(v / v_{0, \mathrm{~S}}\right)=0,2,4$; the dashed line: $\log \left(v / v_{0, \mathrm{~S}}\right)=6$; the dotted line: $\log \left(v / v_{0, \mathrm{~S}}\right)=8$; the dashed and dotted line: $\log \left(v / v_{0, \mathrm{~S}}\right)=9$; the dashed dotted dotted line: $\left.\log \left(v / v_{0, \mathrm{~S}}\right)=10\right)$. 


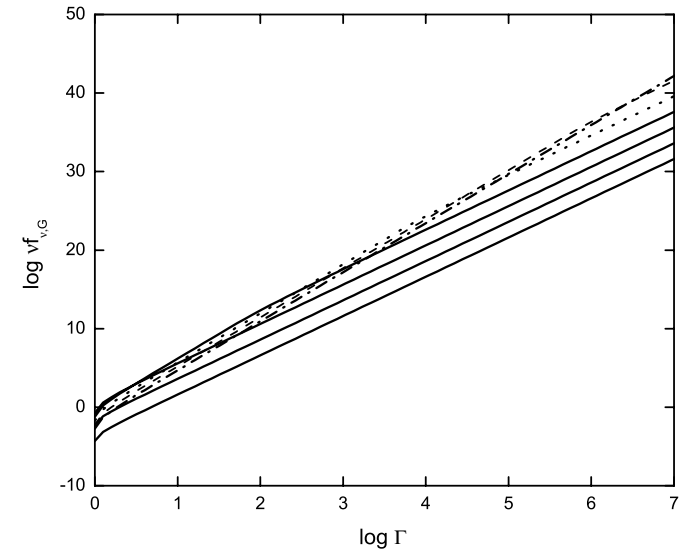

Fig. 5. The plot of $\log \left(v f_{v, \mathrm{G}}\right)-\log \Gamma$ for the radiation bearing the GRB form at various frequencies (the solid lines from the bottom to the top: $\log \left(v / v_{0, \mathrm{G}}\right)=-4,-2,0,2$; the dotted line: $\log \left(v / v_{0, \mathrm{G}}\right)=$ 4; the dashed line: $\log \left(v / v_{0, \mathrm{G}}\right)=6$; the dashed and dotted line: $\left.\log \left(v / v_{0, \mathrm{G}}\right)=8\right)$.
Curves corresponding to $\Gamma=5000,10000$ in Figs. 1-4 and 6-9 in Paper I should be replaced with those with slightly larger values. Since they are plotted in the logarithmic format, the differences are hardly observable, and therefore the revised figures are omitted here.

None of the main conclusions of Paper I, which are presented in the abstract, are affected by this correction. 